\title{
Finite-Time Stability of Large-Scale Systems with Interval Time-Varying Delay in Interconnection
}

\author{
T. La-inchua, ${ }^{1}$ P. Niamsup, ${ }^{1}$ and Xinzhi Liu ${ }^{2}$ \\ ${ }^{1}$ Department of Mathematics, Chiang Mai University, Chiang Mai 50200, Thailand \\ ${ }^{2}$ Department of Applied Mathematics, University of Waterloo, Waterloo, ON, Canada N2L 3G1 \\ Correspondence should be addressed to P. Niamsup; piyapong.n@cmu.ac.th
}

Received 23 June 2016; Revised 9 October 2016; Accepted 1 November 2016; Published 11 January 2017

Academic Editor: Pietro De Lellis

Copyright (c) 2017 T. La-inchua et al. This is an open access article distributed under the Creative Commons Attribution License, which permits unrestricted use, distribution, and reproduction in any medium, provided the original work is properly cited.

\begin{abstract}
We investigate finite-time stability of a class of nonlinear large-scale systems with interval time-varying delays in interconnection. Time-delay functions are continuous but not necessarily differentiable. Based on Lyapunov stability theory and new integral bounding technique, finite-time stability of large-scale systems with interval time-varying delays in interconnection is derived. The finite-time stability criteria are delays-dependent and are given in terms of linear matrix inequalities which can be solved by various available algorithms. Numerical examples are given to illustrate effectiveness of the proposed method.
\end{abstract}

\section{Introduction}

It is well known that several real-world processes often depend on time-delay; namely, the present state depends on the past states. Consequently, time-delay systems have been investigated extensively in the past decades. Time-delay is often the main source of instability and poor performance of the systems (see [1-5]). Therefore, it is important to study time-delay systems, especially those with time-varying delays.

There are two types of stability of time-varying delays systems, namely, delay-dependent and delay-independent ones. The delay-dependent conditions are usually less conservative than delay-independent ones, especially when the time-delays are relatively small. One of the main purposes of delay-dependent stability criteria is to estimate the maximum possible allowable delay bounds.

For stability analysis of time-delay systems, a common approach used is by choosing an appropriate LyapunovKrasovskii functional and then properly estimating upper bounds of its derivative along trajectories of solutions of the system (see $[1,6])$. In order to estimate such upper bounds of derivative of Lyapunov-Krasovskii functional, various mathematical tools have been used such as the Jensen inequality $[3,4,7]$, lower bound lemma for reciprocal convexity [8-11], delay partitioning method [11], and freeweighting matrix variables method $[6,12,13]$. Recently, a new integral inequality which improves Jensen inequality, namely, Wirtinger-based integral inequality, has been proposed in [14]. In [15], a novel integral inequality has been proposed which has been shown to be less conservative than Jensen inequality. In $[16,17]$, some new bounding techniques have been developed and enhanced stability criteria for time-delay systems have been derived using these bounding techniques which are shown to be less conservative than some existing results which used Wirtinger-based or Jensen inequalities. In [18], by using Wirtinger-based integral inequality combined with the reciprocally convex approach, some less conservative stability criteria for time-delay systems are derived.

Large-scale interconnected systems have been the subject of considerable research (see [19-25]), which can be characterized by a large number of variables representing the systems, a strong interaction between subsystem variables, and a complex interaction between subsystems. The large-scale interconnected system can be found in many practical control problems such as transportation systems, electrical power systems, communication systems, and economic systems. The operation of large-scale interconnected systems requires the capability of monitoring and stabilizing in the face of uncertainties, disturbances, failures, and attacks through the 
utilization of internal system states. However, even with the assumption that all the state variables are available for feedback control, the task of effective controlling of a largescale interconnected system using a global (centralized) state feedback controller is still not easy as there is a necessary requirement for information transfer between the subsystems [22].

Generally, studies of dynamical systems are involved with stability analysis which is defined over an infinite-time interval. Nevertheless, in many real-world applications, the main aim is concerned with the behavior of the system over a fixed finite-time interval, for instance, the problem of sending a rocket from the neighborhood of point $A$ to the neighborhood of point $B$ over a fixed time interval [26]. In this example, the concept of finite-time stability (FTS) is proposed. The problem of FTS has been revisited using linear matrix inequality (LMI) technique which allows us to find feasible condition guaranteeing FTS (see [2629]). Some interesting results on stability and stabilization in the context of time-delay systems have been obtained by using the Lyapunov-Krasovskii functional technique (see $[7,11,30])$. Most existing work assumed that the timedelays are either constant or differentiable. In [20, 24], delaydependent sufficient conditions for stability of large-scale system with time-varying delay were studied. However, the approach used there cannot be applied to systems with interval nondifferentiable time-varying delays. Moreover, to the best of our knowledge, there are few results for FTS of large-scale interconnected systems, especially systems with interval nondifferential time-varying delay.

Motivated by the above discussions, we shall derive new FTS criteria for large-scale systems with interval time-varying delays in interconnection. The main contributions of our studies are as follows. (i) The time-delay functions are only required to be continuous but not necessarily differentiable. (ii) By employing an improved integral inequality in [18], we derive new and less conservative FTS for large-scale interconnected systems with time-varying delays in terms of LMIs.

The rest of this paper is organized as follows. Section 2 presents notations, definitions, and auxiliary lemmas required for the proof of the main results. In Section 3, the FTS criteria for large-scale interconnected system with time-varying delays are obtained. Illustrative numerical examples are presented in Section 4. Section 5 gives the conclusion of the paper.

\section{Problem Formulation and Preliminaries}

Under the practical constraint of decentralized information coupled with the facts that the measurement of all the states and the real-time knowledge of the interval time-varying delays are not available, we consider the following nonlinear large-scale interconnected system:

$$
\dot{x}_{i}(t)=A_{i} x_{i}(t)+\sum_{j \neq i, j=1}^{N} A_{i j} x_{j}\left(t-h_{i j}(t)\right)
$$

$$
\begin{array}{cl}
+f_{i}\left(t, x_{i}(t),\left\{x_{j}\left(t-h_{i j}(t)\right)\right\}_{j=1, j \neq i}^{N}\right), & \\
x_{i}(s)=\phi_{i}(s), \quad s \in\left[-h_{2}, 0\right], & t \geq 0
\end{array}
$$

where $x(t)=\left[x_{1}(t), x_{2}(t), \ldots, x_{n}(t)\right]^{T}, x_{i}(t) \in \mathbb{R}^{n_{i}}$ is the state vector, $\phi_{i} \in C^{1}\left(\left[-h_{2}, 0\right], \mathbb{R}^{n_{i}}\right)$ are initial conditions with the norm defined by

$$
\left\|\phi_{i}\right\|=\sup _{s \in\left[-h_{2}, 0\right]}\left\{\left\|\phi_{i}(s)\right\|,\left\|\dot{\phi}_{i}(s)\right\|\right\},
$$

and $h_{i j}(t)$ are time-delay functions which are continuous and satisfy

$$
0 \leq h_{1} \leq h_{i j}(t) \leq h_{2},
$$

for all $t \geq 0$ and all $i, j=1,2, \ldots, N$. The state matrices $A_{i}$ and $A_{i j}$ are of appropriate dimensions. The nonlinear perturbations $f_{i}(\cdot)$ are continuous, satisfying the following conditions.

There exist $a_{i}, a_{i j}>0$ such that

$$
\begin{aligned}
& \left\|f_{i}\left(t, x_{i}(t),\left\{x_{j}\left(t-h_{i j}(t)\right)\right\}_{j=1, j \neq i}^{N}\right)\right\| \\
& \quad \leq a_{i}\left\|x_{i}(t)\right\|+\sum_{j \neq i, j=1}^{N} a_{i j}\left\|x_{j}\left(t-h_{i j}(t)\right)\right\| .
\end{aligned}
$$

For $\phi=\left[\phi_{1}, \phi_{2}, \ldots, \phi_{N}\right]^{T} \in C^{1}\left(\left[-h_{2}, 0\right], \mathbb{R}^{L}\right)$, where $\phi_{i} \epsilon$ $C^{1}\left(\left[-h_{2}, 0\right], \mathbb{R}^{n_{i}}\right)$ and $L=\sum_{i=1}^{N} n_{i}$, we define the norm of $\phi$ as follows:

$$
\|\phi\|_{C^{1}}=\sqrt{\sum_{i=1}^{N}\left\|\phi_{i}\right\|_{C^{1}} .}
$$

The segment of the trajectory of $x(t)$ denoted by $x_{t}$ is defined by $x_{t}=\left\{x(t+s): s \in\left[-h_{2}, 0\right]\right\}$ with the norm defined by $\left\|x_{t}\right\|=\sup _{s \in\left[-h_{2}, 0\right]}\|x(t+s)\|$.

Definition 1 (see [29]). For given positive numbers $c_{1}, c_{2}, T$ and a symmetric positive definite matrix $M$, the nonlinear large-scale system (1) is finite-time stable (FTS) with respect to $\left(c_{1}, c_{2}, T, M\right)$ if the following condition holds:

$$
\begin{aligned}
\sup _{-h_{2} \leq s \leq 0}\left\{\phi(s)^{T} M \phi(s), \dot{\phi}(s)^{T} M \dot{\phi}(s)\right\}<c_{1} & \\
x^{T}(t) M x(t)<c_{2}, & \\
& \forall t \in[0, T] .
\end{aligned}
$$

Proposition 2 (see [22] Schur complement lemma). Given matrices $X, Y, Z$, where $Y=Y^{T}>0$ and $X=X^{T}, X+$ $Z^{T} Y^{-1} Z<0$ if and only if $\left[\begin{array}{cc}X & Z^{T} \\ Z & -Y\end{array}\right]<0$.

Proposition 3 (see [22] Jensen-type integral inequality). For any constant matrix $Z=Z^{T}>0$ and scalar $h>0$ such that the following integrals are well defined, 


$$
\begin{aligned}
& -\int_{t-h}^{t} x(s)^{T} Z x(s) d s \\
& \quad \leq-\frac{1}{h}\left(\int_{t-h}^{t} x(s) d s\right)^{T} Z\left(\int_{t-h}^{t} x(s) d s\right) .
\end{aligned}
$$

Proposition 4 (see [22] Cauchy matrix inequality). For any $x, y \in \mathbb{R}^{n}$ and positive definite matrix $M \in \mathbb{R}^{n \times n}$, we have

$$
2 x^{T} y \leq y^{T} M y+x^{T} M^{-1} x .
$$

Lemma 5 (see [18]). For a given matrix $R>0, h_{m} \leq h(t) \leq$ $h_{M}$ and any appropriate dimension matrix $\chi$ which satisfies $\left[\begin{array}{cc}\bar{R} & \chi \\ \chi^{T} & \bar{R}\end{array}\right] \geq 0$. The following inequality holds for all continuously differentiable function $x(t)$ :

$$
\begin{aligned}
& -\left(h_{M}-h_{m}\right) \int_{t-h_{M}}^{t-h_{m}} \dot{x}(s)^{T} R \dot{x}(s) d s \\
& \leq-\alpha^{T}(t)\left[\begin{array}{cc}
\bar{R} & \chi \\
\chi^{T} & \bar{R}
\end{array}\right] \alpha(t),
\end{aligned}
$$

where

$$
\begin{aligned}
\alpha(t)= & {\left[\alpha_{1}^{T}(t), \alpha_{2}^{T}(t), \alpha_{3}^{T}(t), \alpha_{4}^{T}(t)\right]^{T}, } \\
\alpha_{1}(t)= & x\left(t-h_{m}\right)-x(t-h(t)), \\
\alpha_{2}(t)= & x\left(t-h_{m}\right)+x(t-h(t)) \\
& -\left(\frac{2}{h(t)-h_{m}}\right) \int_{t-h(t)}^{t-h_{m}} x(s) d s, \\
\alpha_{3}(t)= & x(t-h(t))-x\left(t-h_{M}\right), \\
\alpha_{4}(t)= & x(t-h(t))+x\left(t-h_{M}\right) \\
& -\left(\begin{array}{c}
\left.\frac{2}{h_{M}-h(t)}\right) \int_{t-h_{M}}^{t-h(t)} x(s) d s, \\
\bar{R}=
\end{array}\right. \\
& {\left[\begin{array}{cc}
R & 0 \\
* & 3 R
\end{array}\right] . }
\end{aligned}
$$

\section{Finite-Time Stability of Nonlinear Large-Scale Systems}

In this section, we derive FTS criteria for large-scale interconnected system with time-varying delays. For the sake of simplicity, the following notations will be used:

For $i, j, k=1, \ldots, N, j \neq i$ and $k \neq j$, we let

$$
\begin{aligned}
& \Theta_{i, i}^{i}=P_{i} A_{i}+A_{i}^{T} P_{i}+2 Q_{i}-\left(e^{\beta h_{1}}+e^{\beta h_{2}}\right) R_{i}-\beta P \\
& \quad+a_{i} I ; \\
& \Theta_{i, N+1}^{i}=e^{\beta h_{1}} R_{i} ; \\
& \Theta_{i, N+2}^{i}=e^{\beta h_{2}} R_{i} ; \\
& \Theta_{i, N+3}^{i}=A_{i}^{T} P_{i} ;
\end{aligned}
$$

$$
\begin{aligned}
& \Theta_{j, j}^{i}=\left(2+2 a_{j i}\right) I+e^{\beta h_{2}}\left(-8 U_{i}+T_{1 i}+T_{1 i}^{T}+\frac{T_{2 i}}{2}\right. \\
& \left.+\frac{T_{2 i}^{T}}{2}+T_{3 i}+T_{3 i}^{T}-\frac{T_{4 i}}{2}-\frac{T_{4 i}^{T}}{2}\right) ; \\
& \Theta_{j, N+1}^{i}=e^{\beta h_{2}}\left(4 U_{i}-T_{1 i}-T_{2 i}-T_{3 i}-T_{4 i}\right) ; \\
& \Theta_{j, N+2}^{i}=e^{\beta h_{2}}\left(-4 U_{i}-T_{1 i}+\frac{T_{2 i}}{2}+\frac{T_{2 i}^{T}}{2}+T_{3 i}-T_{4 i}\right. \\
& \left.-\frac{T_{4 i}^{T}}{2}\right) ;
\end{aligned}
$$$$
\Theta_{j, N+4}^{i}=e^{\beta h_{2}}\left(3 U_{i}+T_{3 i}^{T}+\frac{T_{4 i}^{T}}{2}\right) ;
$$$$
\Theta_{j, N+5}^{i}=e^{\beta h_{2}}\left(-3 U_{i}+T_{2 i}+\frac{T_{4 i}^{T}}{2}\right) ;
$$$$
\Theta_{N+1, N+1}^{i}=-e^{\beta h_{1}} Q_{i}-e^{\beta h_{1}} R_{i}-4 e^{\beta h_{1}} U_{i},
$$$$
\Theta_{N+1, N+2}^{i}=e^{\beta h_{2}}\left(T_{1 i}-T_{2 i}+T_{3 i}-T_{4 i}\right)
$$$$
\Theta_{N+1, N+4}^{i}=3 e^{\beta h_{2}} U_{i}
$$$$
\Theta_{N+1, N+5}^{i}=e^{\beta h_{2}}\left(T_{2 i}+T_{4 i}\right)
$$$$
\Theta_{N+2, N+2}^{i}=-2 e^{\beta h_{2}} Q_{i}+e^{\beta h_{2}}\left(-R_{i}-4 U_{i}-T_{2 i}-T_{2 i}^{T}\right) ;
$$$$
\Theta_{N+2, N+4}^{i}=e^{\beta h_{2}}\left(T_{3 i}^{T}+\frac{T_{4 i}}{2}\right)
$$$$
\Theta_{N+2, N+5}^{i}=e^{\beta h_{2}}\left(-3 U_{i}+\frac{T_{4 i}}{2}\right),
$$$$
\Theta_{N+3, N+3}^{i}=\left(h_{1}^{2}+h_{2}^{2}\right) R_{i}-2 P_{i}+\left(h_{2}^{2}-h_{1}^{2}\right) U_{i} ;
$$$$
\Theta_{N+4, N+4}^{i}=-3 e^{\beta h_{2}} U_{i}
$$$$
\Theta_{N+4, N+5}^{i}=-e^{\beta h_{2}} T_{4 i},
$$$$
\Theta_{N+5, N+5}^{i}=-3 e^{\beta h_{2}} U_{i}
$$$$
\Theta_{N+5+j, N+5+j}^{i}=-I \text {; }
$$$$
\Theta_{i, N+5+j}^{i}=P_{i} A_{i j}
$$$$
\Theta_{N+5+i, N+5+i}^{i}=-I
$$$$
\Theta_{i, N+5+i}^{i}=\sqrt{\xi_{i}} P_{i}
$$$$
\Theta_{2 N+5+j, 2 N+5+j}^{i}=-I ;
$$$$
\Theta_{N+5,2 N+5+j}^{i}=P_{i} A_{i j} ;
$$

$\Theta_{2 N+5+i, 2 N+5+i}^{i}=-I$ 


$$
\begin{aligned}
& \Theta_{N+5,2 N+5+i}^{i}=\sqrt{\xi_{i}} P_{i} \\
& \zeta_{i}^{T}(t)=\left[\vartheta_{i}^{1}, \ldots, \vartheta_{i}^{N}, x_{i}\left(t-h_{1}\right)^{T}, x_{i}\left(t-h_{2}\right)^{T}, \dot{x}(t)^{T},\right. \\
& \left.\omega_{1}(t)^{T}, \omega_{2}(t)^{T}\right] \\
& \vartheta_{i}^{j}=x_{i}\left(t-h_{j i}(t)\right)^{T}, \quad i \neq j, \quad \vartheta_{i}^{i}=x_{i}^{T}(t) ; \\
& \omega_{1}(t)=\left(\frac{2}{h(t)-h_{1}}\right) \int_{t-h(t)}^{t-h_{1}} x(s) d s ; \\
& \omega_{2}(t)=\left(\frac{2}{h_{2}-h(t)}\right) \int_{t-h_{2}}^{t-h(t)} x(s) d s \\
& \Omega^{i}=\left[\begin{array}{cccccc}
\Omega_{11}^{i} & \Omega_{12}^{i} & \cdot & \cdot & \cdot & \Omega_{1(N+5)}^{i} \\
* & \Omega_{22}^{i} & \cdot & \cdot & \cdot & \Omega_{2(N+5)}^{i} \\
\cdot & \cdot & \cdot & \cdot & \cdot & \cdot \\
* & * & \cdot & \cdot & \cdot & \Omega_{(N+5)(N+5)}^{i}
\end{array}\right] \\
& \Omega_{i, i}^{i}=P_{i} A_{i}+A_{i}^{T} P_{i}+2 Q_{i}-\left(e^{\beta h_{1}}+e^{\beta h_{2}}\right) R_{i}-\beta P+a_{i} I \\
& +\sum_{j \neq i, j=1}^{N} P_{i} A_{i} A_{i}^{T} P_{i}+\xi_{i} P_{i}^{2} \\
& \Omega_{i, N+1}^{i}=e^{\beta h_{1}} R_{i} \\
& \Omega_{i, N+2}^{i}=e^{\beta h_{2}} R_{i} \\
& \Omega_{i, N+3}^{i}=A_{i}^{T} P_{i} \\
& \Omega_{j, j}^{i}=\left(2+2 a_{j i}\right) I+e^{\beta h_{2}}\left(-8 U_{i}+T_{1 i}+T_{1 i}^{T}+\frac{T_{2 i}}{2}\right. \\
& \left.+\frac{T_{2 i}^{T}}{2}+T_{3 i}+T_{3 i}^{T}-\frac{T_{4 i}}{2}-\frac{T_{4 i}^{T}}{2}\right) \\
& \Omega_{j, N+1}^{i}=e^{\beta h_{2}}\left(4 U_{i}-T_{1 i}-T_{2 i}-T_{3 i}-T_{4 i}\right) ; \\
& \Omega_{j, N+2}^{i}=e^{\beta h_{2}}\left(-4 U_{i}-T_{1 i}+\frac{T_{2 i}}{2}+\frac{T_{2 i}^{T}}{2}+T_{3 i}-T_{4 i}\right. \\
& \left.-\frac{T_{4 i}^{T}}{2}\right) \\
& \Omega_{j, N+4}^{i}=e^{\beta h_{2}}\left(3 U_{i}+T_{3 i}^{T}+\frac{T_{4 i}^{T}}{2}\right) ; \\
& \Omega_{j, N+5}^{i}=e^{\beta h_{2}}\left(-3 U_{i}+T_{2 i}+\frac{T_{4 i}^{T}}{2}\right) \\
& \Omega_{N+1, N+1}^{i}=-e^{\beta h_{1}} Q_{i}-e^{\beta h_{1}} R_{i}-4 e^{\beta h_{1}} U_{i} ; \\
& \Omega_{N+1, N+2}^{i}=e^{\beta h_{2}}\left(T_{1 i}-T_{2 i}+T_{3 i}-T_{4 i}\right) ; \\
& \Omega_{N+1, N+4}^{i}=3 e^{\beta h_{2}} U_{i} \text {; }
\end{aligned}
$$

$$
\begin{aligned}
& \Omega_{N+1, N+5}^{i}=e^{\beta h_{2}}\left(T_{2 i}+T_{4 i}\right) ; \\
& \Omega_{N+2, N+2}^{i}=-2 e^{\beta h_{2}} Q_{i}+e^{\beta h_{2}}\left(-R_{i}-4 U_{i}-T_{2 i}-T_{2 i}^{T}\right) ; \\
& \Omega_{N+2, N+4}^{i}=e^{\beta h_{2}}\left(T_{3 i}^{T}+\frac{T_{4 i}}{2}\right) \\
& \Omega_{N+2, N+5}^{i}=e^{\beta h_{2}}\left(-3 U_{i}+\frac{T_{4 i}}{2}\right) ; \\
& \Omega_{N+3, N+3}^{i}=\left(h_{1}^{2}+h_{2}^{2}\right) R_{i}-2 P_{i}+\left(h_{2}^{2}-h_{1}^{2}\right) U_{i} \\
& +\sum_{j \neq i, j=1}^{N} P_{i} A_{i} A_{i}^{T} P_{i}+\xi_{i} P_{i}^{2} ; \\
& \Omega_{N+4, N+4}^{i}=-3 e^{\beta h_{2}} U_{i} \\
& \Omega_{N+4, N+5}^{i}=-e^{\beta h_{2}} T_{4 i} \text {; } \\
& \Omega_{N+5, N+5}^{i}=-3 e^{\beta h_{2}} U_{i} \\
& f_{i}(\cdot)=f_{i}\left(t, x_{i}(t),\left\{x_{j}\left(t-h_{i j}(t)\right)\right\}_{j=1, j \neq i}^{N}\right) \\
& \xi_{i}=a_{i}+\sum_{j \neq i, j=1}^{N} a_{i j} ; \\
& \bar{P}_{i}=M^{-1 / 2} P_{i} M^{-1 / 2} \\
& \alpha_{1}=\min _{i=1, \ldots, N} \lambda_{\min }\left(\bar{P}_{i}\right) \\
& \alpha_{2}=\max _{i=1, \ldots, N}\left\{\lambda_{\max }\left(P_{i}\right)+\beta^{-1} \lambda_{\max }\left(Q_{i}\right)\right. \\
& \left.+\left(h_{1}^{3}+h_{2}^{3}\right) \lambda_{\max }\left(R_{i}\right)+\left(h_{2}-h_{1}\right)^{3} \lambda_{\max }\left(U_{i}\right)\right\} ; \\
& \overline{U_{i}}=\left[\begin{array}{cc}
U_{i} & 0 \\
* & 3 U_{i}
\end{array}\right] \text {; } \\
& \chi_{i}=\left[\begin{array}{cc}
T_{1 i} & T_{2 i} \\
T_{3 i} & T_{4 i}
\end{array}\right]
\end{aligned}
$$

The other matrices $\Theta_{j, k}^{i}$ and $\Omega_{j, k}^{i}$ which do not appear above would be defined to be zero matrices with appropriate dimensions.

Theorem 6. For given positive numbers $c_{1}, c_{2}, T$ and a symmetric positive definite matrix $M$, if there exist symmetric positive definite matrices $P_{i}, Q_{i}, R_{i}, U_{i}$ and matrices $T_{1 i}, \ldots, T_{4 i}$ of appropriate dimensions such that the conditions

$$
\Theta^{i}=\left[\begin{array}{cccccc}
\Theta_{11}^{i} & \Theta_{12}^{i} & \cdot & \cdot & \cdot & \Theta_{1(3 N+5)}^{i} \\
* & \Theta_{22}^{i} & \cdot & \cdot & \cdot & \Theta_{2(3 N+5)}^{i} \\
\cdot & \cdot & \cdot & \cdot & \cdot & \cdot \\
* & * & \cdot & \cdot & \cdot & \Theta_{(3 N+5)(3 N+5)}^{i}
\end{array}\right]<0,
$$

$$
i=1,2, \ldots, N
$$




$$
\frac{\alpha_{2} c_{1}}{\alpha_{1}} \leq c_{2} e^{-\beta T}
$$

hold, then system (1) is FTS with respect to $\left(c_{1}, c_{2}, T, M\right)$.

Proof. We choose the following Lyapunov-Krasovskii functional:

$$
V\left(t, x_{t}\right)=\sum_{i=1}^{N} \sum_{j=1}^{6} V_{i j}\left(t, x_{t}\right)
$$

where

$$
\begin{aligned}
& V_{i 1}\left(t, x_{t}\right)=x_{i}^{T}(t) P_{i} x_{i}(t), \\
& V_{i 2}\left(t, x_{t}\right)=\int_{t-h_{1}}^{t} e^{\beta(t-s)} x^{T}(s) Q_{i} x(s) d s, \\
& V_{i 3}\left(t, x_{t}\right)=\int_{t-h_{2}}^{t} e^{\beta(t-s)} x^{T}(s) Q_{i} x(s) d s, \\
& V_{i 4}\left(t, x_{t}\right)=h_{1} \int_{-h_{1}}^{0} \int_{t+s}^{t} e^{\beta(t-\tau)} \dot{x}_{i}^{T}(\tau) R_{i} \dot{x}_{i}(\tau) d \tau d s, \\
& V_{i 5}\left(t, x_{t}\right)=h_{2} \int_{-h_{2}}^{0} \int_{t+s}^{t} e^{\beta(t-\tau)} \dot{x}_{i}^{T}(\tau) R_{i} \dot{x}_{i}(\tau) d \tau d s, \\
& V_{i 6}\left(t, x_{t}\right) \\
& =\left(h_{2}-h_{1}\right) \int_{-h_{2}}^{-h_{1}} \int_{t+s}^{t} e^{\beta(t-\tau)} \dot{x}_{i}^{T}(\tau) U_{i} \dot{x}_{i}(\tau) d \tau d s .
\end{aligned}
$$

We first show that

$$
\alpha_{1} x^{T}(t) M x(t) \leq V\left(t, x_{t}\right), \quad \forall t: 0 \leq t \leq T .
$$

From

$$
\begin{aligned}
V_{i 1}\left(t, x_{t}\right) & =x_{i}^{T}(t) P_{i} x_{i}(t) \\
& =x_{i}^{T}(t) M^{1 / 2} M^{-1 / 2} P_{i} M^{-1 / 2} M^{1 / 2} x_{i}(t) \\
& =x_{i}^{T}(t) M^{1 / 2} \bar{P}_{i} M^{1 / 2} x_{i}(t) \\
& \geq \lambda_{\min }\left(\bar{P}_{i}\right) x_{i}^{T}(t) M x_{i}(t),
\end{aligned}
$$

we have

$$
V\left(t, x_{t}\right) \geq \alpha_{1} x^{T}(t) M x(t),
$$

where $\alpha_{1}=\min _{i=1, \ldots, N} \lambda_{\min }\left(\bar{P}_{i}\right)$. Thus, (16) holds. Similarly, we obtain the following estimation:

$$
\begin{aligned}
V\left(0, x_{0}\right) & \leq \alpha_{2} \sup _{-h_{2} \leq s \leq 0}\left\{\phi(s)^{T} M \phi(s), \dot{\phi}(s)^{T} M \dot{\phi}(s)\right\} \\
& \leq \alpha_{2} c_{1} .
\end{aligned}
$$

Taking the derivative of $V\left(t, x_{t}\right)$ with respect to $t$ along the trajectory of solution of the system (1), we obtain

$$
\begin{aligned}
& \dot{V}_{i 1}(\cdot)=2 x_{i}^{T}(t) P_{i} \dot{x}(t)=2 x_{i}^{T}(t) \\
& \cdot P_{i}\left[A_{i} x_{i}(t)+\sum_{j \neq i, j=1}^{N} A_{i j} x_{j}\left(t-h_{i j}(t)\right)+f_{i}(\cdot)\right], \\
& \dot{V}_{i 2}(\cdot)=x_{i}^{T}(t) Q_{i} x_{i}(t)-e^{\beta h_{1}} x_{i}^{T}\left(t-h_{1}\right) Q_{i} x_{i}\left(t-h_{1}\right) \\
& \quad+\beta V_{i 2}(\cdot), \\
& \dot{V}_{i 3}(\cdot)=x_{i}^{T}(t) Q_{i} x_{i}(t)-e^{\beta h_{2}} x_{i}^{T}\left(t-h_{1}\right) Q_{i} x_{i}\left(t-h_{2}\right) \\
& \quad+\beta V_{i 3}(\cdot), \\
& \dot{V}_{i 4}(\cdot) \leq h_{1}^{2} \dot{x}_{i}^{T}(t) R_{i} \dot{x}_{i}(t) \\
& \quad-h_{1} e^{\beta h_{1}} \int_{t-h_{1}}^{t} \dot{x}_{i}^{T}(s) R_{i} \dot{x}_{i}(s) d s+\beta V_{i 4}(\cdot), \\
& \dot{V}_{i 5}(\cdot) \leq h_{2}^{2} \dot{x}_{i}^{T}(t) R_{i} \dot{x}_{i}(t) \\
& -h_{2} e^{\beta h_{2}} \int_{t-h_{2}}^{t} \dot{x}_{i}^{T}(s) R_{i} \dot{x}_{i}(s) d s+\beta V_{i 5}(\cdot), \\
& \dot{V}_{i 6}(\cdot) \leq\left(h_{2}-h_{1}\right)^{2} \dot{x}_{i}^{T}(t) U_{i} \dot{x}_{i}(t)-\left(h_{2}-h_{1}\right) \\
& \cdot e^{\beta h_{2}} \int_{t-h_{2}}^{t-h_{1}} \dot{x}_{i}^{T}(s) U_{i} \dot{x}_{i}(s) d s+\beta V_{i 6}(\cdot) .
\end{aligned}
$$

From Proposition 4, we have

$$
\begin{aligned}
2 x_{i}^{T}(t) & P_{i} \sum_{j \neq i, j=1}^{N} A_{i j} x_{j}\left(t-h_{i j}(t)\right) \\
\leq & \sum_{j \neq i, j=1}^{N} x_{i}(t)^{T} P_{i} A_{i} A_{i}^{T} P_{i} x_{i}(t) \\
& +\sum_{j \neq i, j=1}^{N} x_{i}\left(t-h_{i j}(t)\right)^{T} x_{i}\left(t-h_{i j}(t)\right) .
\end{aligned}
$$

It follows from (4) and Proposition 4 that

$$
\begin{aligned}
& 2 x_{i}^{T}(t) P_{i} f_{i}(\cdot) \leq 2\left\|x_{i}^{T} P_{i}\right\| \\
& \quad\left\{a_{i}\left\|x_{i}(t)\right\|+\sum_{j \neq i, j=1}^{N} a_{i j}\left\|x_{j}\left(t-h_{i j}(t)\right)_{i}\right\|\right\}, \\
& \quad \leq \xi_{i}\left\|x_{i}(t)^{T} P_{i}\right\|^{2}+a_{i}\left\|x_{i}(t)\right\|^{2} \\
& \quad+\sum_{j \neq i, j=1}^{N} a_{i j}\left\|x_{j}\left(t-h_{i j}(t)\right)\right\|^{2} .
\end{aligned}
$$


From (22), we have

$$
\begin{aligned}
\dot{V}_{i 1}(\cdot) \leq & x_{i}^{T}(t)\left[P_{i} A_{i}+A_{i}^{T} P_{i}\right] x_{i}(t) \\
& +\sum_{j \neq i, j=1}^{N} x_{i}(t)^{T} P_{i} A_{i} A_{i}^{T} P_{i} x_{i}(t) \\
& +\sum_{j \neq i, j=1}^{N} x_{i}\left(t-h_{i j}(t)\right)^{T} x_{i}\left(t-h_{i j}(t)\right) \\
& +\xi_{i}\left\|x_{i}(t)^{T} P_{i}\right\|^{2}+a_{i}\left\|x_{i}(t)\right\|^{2} \\
& +\sum_{j \neq i, j=1}^{N} a_{i j}\left\|x_{j}\left(t-h_{i j}(t)\right)\right\|^{2} .
\end{aligned}
$$

From Proposition 3 and Newton-Leibniz formula, we obtain

$$
\begin{aligned}
& -h_{i} e^{-\beta h_{k}} \int_{t-h_{k}}^{t} \dot{x}_{i}^{T}(s) R_{i} \dot{x}_{i}(s) d s \\
& \leq-\left[\int_{t-h_{k}}^{t} \dot{x}_{i}(s) d s\right]^{T} R_{i}\left[\int_{t-h_{k}}^{t} \dot{x}_{i}(s) d s\right] \\
& =-\left[x_{i}(t)-x_{i}\left(t-h_{k}\right)\right]^{T} R\left[x_{i}(t)-x_{i}\left(t-h_{k}\right)\right],
\end{aligned}
$$

for $k=1,2$. It follows that

$$
\begin{gathered}
\dot{V}_{i 4}(\cdot) \leq h_{1}^{2} \dot{x}_{i}^{T}(t) R_{i} \dot{x}_{i}(t) \\
-h_{1} e^{\beta h_{1}}\left[x_{i}(t)-x_{i}\left(t-h_{1}\right)\right]^{T} \\
\cdot R\left[x_{i}(t)-x_{i}\left(t-h_{1}\right)\right]+\beta V_{i 4}(\cdot), \\
\dot{V}_{i 5}(\cdot) \leq h_{2}^{2} \dot{x}_{i}^{T}(t) R_{i} \dot{x}_{i}(t) \\
-h_{2} e^{\beta h_{2}}\left[x_{i}(t)-x_{i}\left(t-h_{2}\right)\right]^{T} \\
\cdot R\left[x_{i}(t)-x_{i}\left(t-h_{2}\right)\right]+\beta V_{i 5}(\cdot) .
\end{gathered}
$$

From Lemma 5, we have

$$
\begin{gathered}
-\left(h_{2}-h_{1}\right) \int_{t-h_{2}}^{t-h_{1}} \dot{x}_{i}^{T}(s) U_{i} \dot{x}_{i}(s) d s \\
\leq-\alpha^{T}(t)\left[\begin{array}{ll}
\overline{U_{i}} & \chi_{i} \\
\chi_{i}^{T} & \overline{U_{i}}
\end{array}\right] \alpha(t) .
\end{gathered}
$$

Thus, from (26), we obtain

$$
\begin{aligned}
\dot{V}_{i 6}(\cdot) \leq & \left(h_{2}-h_{1}\right)^{2} \dot{x}_{i}^{T}(t) U_{i} \dot{x}_{i}(t)+\beta V_{i 6}(\cdot) \\
& -e^{\beta h_{2}} \alpha^{T}(t)\left[\begin{array}{cc}
\overline{U_{i}} & \chi_{i} \\
\chi_{i}^{T} & \overline{U_{i}}
\end{array}\right] \alpha(t) .
\end{aligned}
$$

Hence, we obtain the estimation of $\dot{V}\left(t, x_{t}\right)$ as

$$
\begin{aligned}
\dot{V}\left(t, x_{t}\right) \leq & x_{i}^{T}(t)\left[P_{i} A_{i}+A_{i}^{T} P_{i}\right] x_{i}(t) \\
+ & \sum_{j \neq i, j=1}^{N} x_{i}(t)^{T} P_{i} A_{i} A_{i}^{T} P_{i} x_{i}(t) \\
+ & \sum_{j \neq i, j=1}^{N} x_{i}\left(t-h_{i j}(t)\right)^{T} x_{i}\left(t-h_{i j}(t)\right) \\
+ & \xi_{i}\left\|x_{i}(t)^{T} P_{i}\right\|^{2}+a_{i}\left\|x_{i}(t)\right\|^{2} \\
+ & \sum_{j \neq i, j=1}^{N} a_{i j}\left\|x_{j}\left(t-h_{i j}(t)\right)\right\|^{2}+x_{i}^{T}(t) \\
& \cdot Q_{i} x_{i}(t)-e^{\beta h_{1}} x_{i}^{T}\left(t-h_{1}\right) Q_{i} x_{i}\left(t-h_{1}\right) \\
+ & x_{i}^{T}(t) Q_{i} x_{i}(t)-e^{\beta h_{2}} x_{i}^{T}\left(t-h_{1}\right) \\
& \cdot Q_{i} x_{i}\left(t-h_{2}\right)+h_{1}^{2} \dot{x}_{i}^{T}(t) R_{i} \dot{x}_{i}(t) \\
& -h_{1} e^{\beta h_{1}}\left[x_{i}(t)-x_{i}\left(t-h_{1}\right)\right]^{T} \\
& \cdot R\left[x_{i}(t)-x_{i}\left(t-h_{1}\right)\right]+h_{2}^{2} \dot{x}_{i}^{T}(t) R_{i} \dot{x}_{i}(t) \\
& -h_{2} e^{\beta h_{2}}\left[x_{i}(t)-x_{i}\left(t-h_{2}\right)\right]^{T} \\
& \cdot R\left[x_{i}(t)-x_{i}\left(t-h_{2}\right)\right]+\left(h_{2}-h_{1}\right)^{2} \dot{x}_{i}(t)-e^{\beta h_{2}} \alpha^{T}(t) \\
& \left.\chi_{i}\right](t) . \\
& \\
& \\
& \\
& \\
& \\
&
\end{aligned}
$$

From (1) and Proposition 4, we get

$$
\begin{aligned}
0= & -2 \dot{x}(t)^{T} P_{i}\left[\dot{x}(t)-A_{i} x_{i}(t)\right. \\
& \left.-\sum_{j \neq i, j=1}^{N} A_{i j} x_{j}\left(t-h_{i j}(t)\right)-f_{i}(\cdot)\right] \leq-2 \dot{x}(t)^{T} \\
& \cdot P_{i}\left[\dot{x}(t)-A_{i} x_{i}(t)\right] \\
& +\sum_{j \neq i, j=1}^{N} \dot{x}_{i}(t)^{T} P_{i} A_{i} A_{i}^{T} P_{i} \dot{x}_{i}(t) \\
& +\sum_{j \neq i, j=1}^{N} x_{i}\left(t-h_{i j}(t)\right)^{T} x_{i}\left(t-h_{i j}(t)\right) \\
& +\xi_{i}\left\|\dot{x}_{i}(t)^{T} P_{i}\right\|^{2}+a_{i}\left\|x_{i}(t)\right\|^{2} \\
& +\sum_{j \neq i, j=1}^{N} a_{i j}\left\|x_{j}\left(t-h_{i j}(t)\right)\right\|^{2} .
\end{aligned}
$$


Adding inequality (29) into the left-hand side of (28) and from the fact that

$$
\begin{aligned}
& \sum_{i=1}^{N} \sum_{j \neq i, j=1}^{N} x_{j}\left(t-h_{i j}(t)\right)^{T} x_{j}\left(t-h_{i j}(t)\right) \\
& =\sum_{j=1}^{N} \sum_{i \neq j, i=1}^{N} x_{i}\left(t-h_{j i}(t)\right)^{T} x_{i}\left(t-h_{j i}(t)\right) \\
& =\sum_{i=1}^{N}\left[\sum_{j \neq i, j=1}^{N} x_{i}\left(t-h_{j i}(t)\right)^{T} x_{i}\left(t-h_{j i}(t)\right)\right], \\
& \sum_{i=1}^{N} \sum_{j \neq i, j=1}^{N} a_{i j}\left\|x_{j}\left(t-h_{i j}(t)\right)\right\|^{2} \\
& =\sum_{i=1}^{N} \sum_{j \neq i, j=1}^{N} a_{j i}\left\|x_{i}\left(t-h_{j i}(t)\right)\right\|^{2},
\end{aligned}
$$

we have

$$
\dot{V}\left(t, x_{t}\right)-\beta V\left(t, x_{t}\right) \leq \sum_{i=1}^{N} \zeta_{i}^{T}(t) \Omega^{i} \zeta_{i}(t) .
$$

From assumption (12), it follows from Proposition 2 that $\Omega^{i}<$ $0, \forall i=1, \ldots, N$.

Therefore, we have

$$
\dot{V}\left(t, x_{t}\right)-\beta V\left(t, x_{t}\right)<0, \quad \forall t: 0 \leq t \leq T .
$$

Multiply both sides of (32) by $e^{-\beta t}$; we obtain

$$
e^{-\beta t} \dot{V}\left(t, x_{t}\right)-\beta e^{-\beta t} \beta V\left(t, x_{t}\right)<0, \quad \forall t: 0 \leq t \leq T .
$$

Integrating both sides of (33) from 0 to $t$, we have

$$
e^{-\beta t} V\left(t, x_{t}\right)<V\left(0, x_{0}\right), \quad \forall t: 0 \leq t \leq T .
$$

Hence, from (16) and (19), it follows that

$$
\alpha_{1} e^{-\beta t} x(t)^{T} M x(t)<e^{-\beta t} V\left(t, x_{t}\right) \leq \alpha_{2} c_{1} .
$$

From (13), we have

$$
x^{T} M x(t)<\frac{\alpha_{2} c_{1}}{\alpha_{1}} e^{\beta t} \leq c_{2}, \quad \forall t: 0 \leq t \leq T .
$$

Therefore, system (1) is FTS with respect to $\left(c_{1}, c_{2}, T, M\right)$.

Remark 7. The obtained FTS criteria (12) are delay-dependent and are formulated in terms of solutions of LMIs which can be easily solved by various available algorithms such as MATLAB LMI Toolbox [31].

Remark 8. In Theorem 6, the chosen Lyapunov-Krasovskii functional depends only on the lower and upper bounds of time-delay functions to remove restrictions on the differentiability of time-delay functions. In several existing results on stability of large-scale systems, for example, in [20, 24], the restrictions on the differentiability of time-delay functions are required. As a result, the methods proposed in [20,24] are not applicable to system (1).
Remark 9. In $[6,12,13]$, the free-weighting matrix variables have been introduced to obtain stability conditions for timedelay systems. On the other hand, we have not introduced free-weighting matrix variables in order to derive stability criteria which results in less matrix variables regarding computational burdens.

Remark 10. In Theorem 6, we have used Proposition 3 (Jensen inequality) to estimate some integrals and to derive FTS criterion for large-scale interconnected systems with time-varying delays. Nonetheless, Proposition 3 is rather restrictive and further improvement can be made if new refined Jensen-based inequalities reported in the recent literature, for example, in $[16,17]$, are used.

Remark 11. In (1), if $f_{i}(\cdot) \equiv 0$, then we have the following linear large-scale system with interval time-varying delays in interconnection:

$$
\begin{aligned}
& \dot{x}_{i}(t)=A_{i} x_{i}(t)+\sum_{j \neq i, j=1}^{N} A_{i j} x_{j}\left(t-h_{i j}(t)\right), \quad t \geq 0, \\
& x_{i}(s)=\phi_{i}(s), \quad s \in\left[-h_{2}, 0\right] .
\end{aligned}
$$

Then, from Theorem 6, we obtain FTS criteria for linear largescale system (37) as in the following corollary.

Corollary 12. For given $\beta, T>0, c_{2}>c_{1}>0$, and let $M$ be a symmetric positive definite matrix. If there exist symmetric positive definite matrices $P_{i}, Q_{i}, R_{i}, U_{i}$ and matrices $T_{1 i}, \ldots, T_{4 i}$ of appropriate dimensions such that the conditions

$$
\begin{aligned}
& \Gamma^{i}=\left[\begin{array}{cccccc}
\Gamma_{11}^{i} & \Gamma_{12}^{i} & \cdot & \cdot & \Gamma_{1(3 N+5)}^{i} \\
* & \Gamma_{22}^{i} & \cdot & \cdot & \cdot & \Gamma_{2(3 N+5)}^{i} \\
\cdot & \cdot & \cdot & \cdot & \cdot & \cdot \\
* & * & \cdot & \cdot & \cdot & \Gamma_{(3 N+5)(3 N+5)}^{i}
\end{array}\right]<0, \\
& \frac{\alpha_{2} c_{1}}{\alpha_{1}} \leq c_{2} e^{-\beta T}
\end{aligned}
$$

hold, where, for $i, j, k=1, \ldots, N, j \neq i$ and $k \neq j$, we have

$$
\begin{aligned}
& \Gamma_{i, i}^{i}=P_{i} A_{i}+A_{i}^{T} P_{i}+2 Q_{i}-\left(e^{\beta h_{1}}+e^{\beta h_{2}}\right) R_{i}-\beta P ; \\
& \Gamma_{i, N+1}^{i}=e^{\beta h_{1}} R_{i} ; \\
& \Gamma_{i, N+2}^{i}=e^{\beta h_{2}} R_{i} ; \\
& \Gamma_{i, N+3}^{i}=A_{i}^{T} P_{i} ; \\
& \Gamma_{j, j}^{i}=2 I+e^{\beta h_{2}}\left(-8 U_{i}+T_{1 i}+T_{1 i}^{T}+\frac{T_{2 i}}{2}+\frac{T_{2 i}^{T}}{2}+T_{3 i}\right. \\
& \left.\quad+T_{3 i}^{T}-\frac{T_{4 i}}{2}-\frac{T_{4 i}^{T}}{2}\right) ; \\
& \Gamma_{j, N+1}^{i}=e^{\beta h_{2}}\left(4 U_{i}-T_{1 i}-T_{2 i}-T_{3 i}-T_{4 i}\right) ;
\end{aligned}
$$




$$
\begin{aligned}
& \Gamma_{j, N+2}^{i}=e^{\beta h_{2}}\left(-4 U_{i}-T_{1 i}+\frac{T_{2 i}}{2}+\frac{T_{2 i}^{T}}{2}+T_{3 i}-T_{4 i}\right. \\
& \left.-\frac{T_{4 i}^{T}}{2}\right) \\
& \Gamma_{j, N+4}^{i}=e^{\beta h_{2}}\left(3 U_{i}+T_{3 i}^{T}+\frac{T_{4 i}^{T}}{2}\right) ; \\
& \Gamma_{j, N+5}^{i}=e^{\beta h_{2}}\left(-3 U_{i} T_{2 i}+\frac{T_{4 i}^{T}}{2}\right) ; \\
& \Gamma_{N+1, N+1}^{i}=-e^{\beta h_{1}} Q_{i}-e^{\beta h_{1}} R_{i}-4 e^{\beta h_{1}} U_{i} \\
& \Gamma_{N+1, N+2}^{i}=e^{\beta h_{2}}\left(T_{1 i}-T_{2 i}+T_{3 i}-T_{4 i}\right) ; \\
& \Gamma_{N+1, N+4}^{i}=3 e^{\beta h_{2}} U_{i} \\
& \Gamma_{N+1, N+5}^{i}=e^{\beta h_{2}}\left(T_{2 i}+T_{4 i}\right) ; \\
& \Gamma_{N+2, N+2}^{i}=-2 e^{\beta h_{2}} Q_{i}+e^{\beta h_{2}}\left(-R_{i}-4 U_{i}-T_{2 i}-T_{2 i}^{T}\right) ; \\
& \Gamma_{N+2, N+4}^{i}=e^{\beta h_{2}}\left(T_{3 i}^{T}+\frac{T_{4 i}}{2}\right) \\
& \Gamma_{N+2, N+5}^{i}=e^{\beta h_{2}}\left(-3 U_{i}+\frac{T_{4 i}}{2}\right) ; \\
& \Gamma_{N+3, N+3}^{i}=\left(h_{1}^{2}+h_{2}^{2}\right) R_{i}-2 P_{i}+\left(h_{2}^{2}-h_{1}^{2}\right) U_{i} ; \\
& \Gamma_{N+4, N+4}^{i}=-3 e^{\beta h_{2}} U_{i} \text {; } \\
& \Gamma_{N+4, N+5}^{i}=-e^{\beta h_{2}} T_{4 i} ; \\
& \Gamma_{N+5, N+5}^{i}=-3 e^{\beta h_{2}} U_{i} \text {; } \\
& \Gamma_{N+5+j, N+5+j}^{i}=-I ; \\
& \Gamma_{i, N+5+j}^{i}=P_{i} A_{i j} ; \\
& \Gamma_{2 N+5+j, 2 N+5+j}^{i}=-I \text {; } \\
& \Gamma_{N+5,2 N+5+j}^{i}=P_{i} A_{i j} ;
\end{aligned}
$$

and other matrices $\Gamma_{j, k}^{i}$ which do not appear above would be defined to be zero matrices with appropriate dimensions, then system (37) is FTS with respect to $\left(c_{1}, c_{2}, T, M\right)$.

\section{Numerical Examples}

In this section, we provide numerical examples to illustrate the effectiveness of our theoretical results.

Example 1. Consider the following nonlinear large-scale systems (1) composed of two machine subsystems which are studied in [23]:

$$
\begin{aligned}
\dot{x}_{1}(t)= & A_{1} x_{1}(t)+A_{12} x_{2}\left(t-h_{12}(t)\right) \\
& +f_{1}\left(t, x_{1}(t), x_{2}\left(t-h_{12}(t)\right)\right), \\
x_{1}(s)= & \phi_{1}(s), \quad s \in\left[-h_{2}, 0\right], \\
\dot{x}_{2}(t)= & A_{2} x_{2}(t)+A_{21} x_{2}\left(t-h_{21}(t)\right) \\
& +f_{2}\left(t, x_{2}(t), x_{1}\left(t-h_{21}(t)\right)\right), \\
x_{2}(s)= & \phi_{2}(s), \quad s \in\left[-h_{2}, 0\right],
\end{aligned}
$$

where the absolute rotor angle and angular velocity of the machine in each subsystem are denoted by $x_{1}=\left[\begin{array}{ll}x_{11} & x_{12}\end{array}\right]^{T}$ and $x_{2}=\left[\begin{array}{ll}x_{21} & x_{22}\end{array}\right]^{T}$, respectively. The $i$ th subsystem state matrices $A_{i}$, the nonlinear perturbations $f_{i}(\cdot)$, the modulus of the transfer admittance $A_{i j}$, and the time-varying delays $h_{i j}(t)$ between the two machines in the subsystems are given by

$$
\begin{aligned}
A_{1} & =\left[\begin{array}{cc}
-1.2 & 0.1 \\
0.2 & -1.3
\end{array}\right], \\
A_{12} & =\left[\begin{array}{cc}
0.1 & 0 \\
0 & 0.1
\end{array}\right], \\
A_{2} & =\left[\begin{array}{cc}
-1.1 & 0.2 \\
0.1 & -1
\end{array}\right], \\
A_{21} & =\left[\begin{array}{cc}
0.1 & 0 \\
0 & 0.2
\end{array}\right], \\
h_{12}(t) & =0.1+0.4|\sin (t)|, \\
h_{21}(t) & =0.1+0.4\left|\sin ^{2}(t)\right|, \\
f_{1}(\cdot) & =0.1\left[\begin{array}{c}
\sqrt{x_{11}(t)^{2}+x_{21}\left(t-h_{12}\right)^{2}} \\
\sqrt{x_{12}(t)^{2}+x_{22}\left(t-h_{12}\right)^{2}}
\end{array}\right], \\
f_{2}(\cdot) & =0.1\left[\begin{array}{c}
\sqrt{x_{21}(t)^{2}+x_{11}\left(t-h_{21}\right)^{2}} \\
\sqrt{x_{22}(t)^{2}+x_{12}\left(t-h_{21}\right)^{2}}
\end{array}\right] .
\end{aligned}
$$

By using LMI Control Toolbox, LMIs (12) and (13) are feasible with solutions given by

$$
\begin{aligned}
& h_{1}=0.1, \\
& h_{2}=0.5, \\
& \beta=0.01, \\
& c_{1}=0.5, \\
& c_{2}=4, \\
& T=10, \\
& M=\left[\begin{array}{ll}
1 & 0 \\
0 & 1
\end{array}\right],
\end{aligned}
$$




$$
\begin{aligned}
& P_{1}=\left[\begin{array}{ll}
0.6660 & 0.0726 \\
0.0726 & 0.6042
\end{array}\right], \\
& P_{2}=\left[\begin{array}{ll}
0.2148 & 0.0140 \\
0.0140 & 0.2001
\end{array}\right] \text {, } \\
& Q_{1}=\left[\begin{array}{ll}
0.1426 & 0.0388 \\
0.0388 & 0.1737
\end{array}\right] \text {, } \\
& Q_{2}=\left[\begin{array}{ll}
0.9293 & 0.0998 \\
0.0998 & 0.8446
\end{array}\right] \text {, } \\
& R_{1}=\left[\begin{array}{ll}
1.4417 & 0.0525 \\
0.0525 & 1.3487
\end{array}\right] \text {, } \\
& R_{2}=\left[\begin{array}{ll}
1.9777 & 0.2090 \\
0.2090 & 1.7997
\end{array}\right] \text {, } \\
& U_{1}=\left[\begin{array}{ll}
0.6081 & 0.0421 \\
0.0421 & 0.5719
\end{array}\right] \text {, } \\
& U_{2}=\left[\begin{array}{ll}
2.5098 & 0.2700 \\
0.2700 & 2.2802
\end{array}\right] \text {, } \\
& T_{11}=\left[\begin{array}{ll}
1.1797 & 0.0822 \\
0.0892 & 1.0967
\end{array}\right] \text {, } \\
& T_{12}=\left[\begin{array}{ll}
2.8450 & 0.2867 \\
0.2868 & 2.6001
\end{array}\right] \text {, } \\
& T_{21}=\left[\begin{array}{ll}
1.1520 & 0.0629 \\
0.0660 & 1.0956
\end{array}\right], \\
& T_{22}=\left[\begin{array}{ll}
2.9639 & 0.2834 \\
0.2835 & 2.7224
\end{array}\right], \\
& T_{31}=\left[\begin{array}{ll}
-1.5532 & -0.0755 \\
-0.0792 & -1.4895
\end{array}\right] \text {, } \\
& T_{32}=\left[\begin{array}{ll}
-3.5433 & -0.3274 \\
-0.3273 & -3.2645
\end{array}\right] \text {, } \\
& T_{41}=\left[\begin{array}{ll}
0.2621 & 0.0168 \\
0.0163 & 0.2459
\end{array}\right] \text {, } \\
& T_{42}=\left[\begin{array}{ll}
0.1844 & 0.0138 \\
0.0138 & 0.1729
\end{array}\right] \text {. }
\end{aligned}
$$

Thus, from Theorem 6, large-scale interconnected system is FTS with respect to $\left(c_{1}, c_{2}, T, M\right)$. The trajectories of solutions of the two subsystems are given in Figure 1. In Figure 2, it is shown that if the initial condition satisfies

$$
\sup _{-h_{2} \leq s \leq 0}\left\{\phi(s)^{T} M \phi(s), \dot{\phi}(s)^{T} M \dot{\phi}(s)\right\}<c_{1},
$$

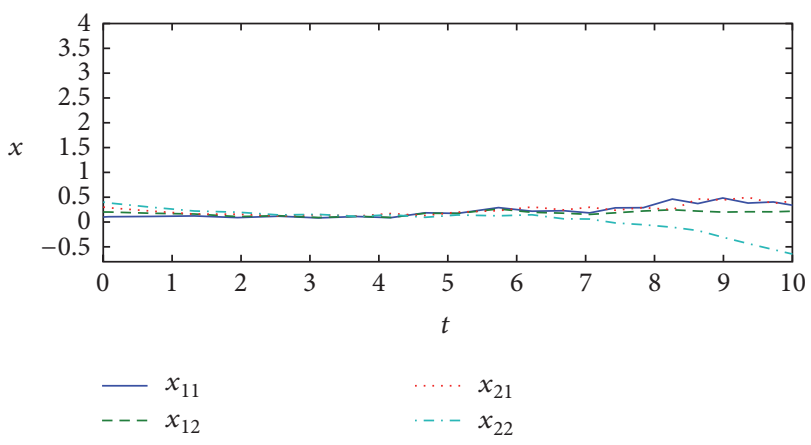

FIGURE 1: The trajectory of solutions of large-scale systems (40).

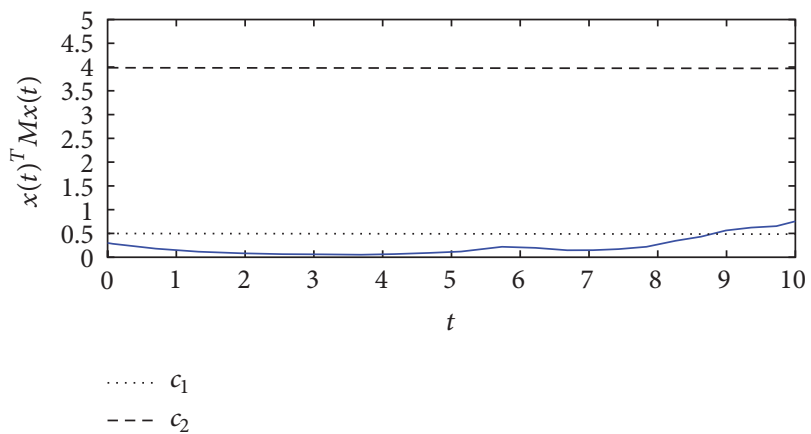

Figure 2: Bounds of $x(t)^{T} M x(t)$ where $x(t)$ is the state of large-scale systems (40).

then $x^{T}(t) M x(t)<c_{2}, \forall t \in[0,10]$, where $x_{1}(0)=\left[\begin{array}{ll}0.1 & 0.2\end{array}\right]^{T}$, $x_{2}(0)=\left[\begin{array}{ll}0.3 & 0.4\end{array}\right]^{T}, \phi_{1}(s)=\left[\begin{array}{ll}0.1 e^{s} & 0.2 e^{s}\end{array}\right]^{T}$, and $\phi_{2}(s)=$ $\left[\begin{array}{ll}0.3 e^{s} & 0.4 e^{s}\end{array}\right]^{T}, s \in\left[-h_{2}, 0\right)$.

Example 2. Consider the following bidirectional associative memory (BAM) neural networks which are studied in [32]:

$$
\begin{aligned}
\dot{x}(t)= & -A x(t)+C f(y(t))+E f(y(t-h(t))) \\
& +u(t), \\
z_{1}(t)= & f(y(t))+f(y(t-h(t)))+u(t), \\
\dot{y}(t)= & -B y(t)+D g(x(t))+F g(x(t-\tau(t))) \\
& +v(t) \\
z_{2}(t)= & g(x(t))+g(x(t-\tau(t)))+v(t),
\end{aligned}
$$

$$
t \geq t_{0}
$$

where $A=\left[\begin{array}{cc}1.8 & 0 \\ 0 & 2.2\end{array}\right], C=\left[\begin{array}{ll}0 & 0 \\ 0 & 0\end{array}\right], E=\left[\begin{array}{cc}0.1 & 0 \\ 0 & 0.1 \\ 0 & 0.2\end{array}\right], B=\left[\begin{array}{cc}2.5 & 0 \\ 0 & 2.2\end{array}\right], D=$ $\left[\begin{array}{ll}0 & 0 \\ 0 & 0\end{array}\right], F=\left[\begin{array}{ll}1 & 0 \\ 0 & 1\end{array}\right], L=\left[\begin{array}{cc}0.1 & 0 \\ 0 & 0.1\end{array}\right], H=\left[\begin{array}{ccc}1 & 0 \\ 0 & 1\end{array}\right], f_{i}(s)=s, g_{i}(s)=$ $0.1 s,(i=1,2)$, and $h(t)$ and $\tau(t)$ are time-varying delays which are continuous but not necessarily differentiable. In Table 1, we give a comparison for maximum allowable upper bounds of time-varying delay, $h_{1} \leq h(t) \leq h_{2}$ and $h_{1} \leq$ $\tau(t) \leq h_{2}$, which are obtained from Theorem 3.1 in [32] and our main result, Theorem 6 . 
TABLE 1: Maximum allowable upper bounds $h_{2}$ of the time-varying delay for different values of the lower bounds $h_{1}$ in example of [32].

\begin{tabular}{lcc}
\hline$h_{1}$ & Theorem 3.1 in [32] & Theorem 6 \\
\hline 0.1 & 9.4999 & 9.5167 \\
0.5 & 10.2013 & 12.0028 \\
0.75 & 10.2068 & 11.7519 \\
1 & 10.2110 & 11.4846 \\
\hline
\end{tabular}

\section{Conclusion}

In this paper, new delay-dependent FTS criteria of nonlinear large-scale interconnected systems with time-varying delay have been derived in terms of solutions of LMIs which could be solved by various available algorithms. By choosing an appropriate Lyapunov-Krasovskii functional and then by using an improved integral inequality, it has been shown by two numerical examples that the obtained FTS criteria are effective and less conservative than some existing results.

\section{Notations}

$\begin{array}{ll}\mathbb{R}^{n}: & \text { The } n \text {-dimensional Euclidean space } \\ \mathbb{R}^{n \times n}: & \text { The set of all } n \times n \text { real matrices } \\ \mathbb{N}: & \text { The set of all positive integers } \\ \operatorname{diag}\{\cdot\}: & \text { The block diagonal matrix } \\ A^{T}: & \text { The transposition of matrix } A \\ A \geq 0(A>0): & A \text { is a semipositive definite matrix } \\ & \text { (positive) } \\ A \geq B(A>B): & A-B \geq 0(A-B>0) \\ \lambda_{\max }(A): & \max \{\operatorname{Re}(\lambda): \lambda \text { is eigenvalue of } A\} \\ \lambda_{\min }(A): & \min \{\operatorname{Re}(\lambda): \lambda \text { is eigenvalue of } A\} \\ \|\cdot\|: & \text { The usual Euclidean norm (or the induced } \\ & \text { matrix norm). }\end{array}$

\section{Competing Interests}

The authors declare that they have no competing interests.

\section{Acknowledgments}

T. La-inchua is supported by Thai Government Scholarships in the Area of Science and Technology (Ministry of Science and Technology). P. Niamsup is supported by Chiang Mai University, Chiang Mai, Thailand.

\section{References}

[1] T. Botmart, P. Niamsup, and V. N. Phat, "Delay-dependent exponential stabilization for uncertain linear systems with interval non-differentiable time-varying delays," Applied Mathematics and Computation, vol. 217, no. 21, pp. 8236-8247, 2011.

[2] J. Liu, X. Liu, and W.-C. Xie, "Delay-dependent robust control for uncertain switched systems with time-delay," Nonlinear Analysis: Hybrid Systems, vol. 2, no. 1, pp. 81-95, 2008.

[3] D. Wang and W. Wang, "Delay-dependent robust exponential stabilization for uncertain systems with interval time-varying delays," Journal of Control Theory and Applications, vol. 7, no. 3, pp. 257-263, 2009.
[4] W. Zhang, X.-S. Cai, and Z.-Z. Han, "Robust stability criteria for systems with interval time-varying delay and nonlinear perturbations," Journal of Computational and Applied Mathematics, vol. 234, no. 1, pp. 174-180, 2010.

[5] X. Zhao, L. Zhang, and P. Shi, "Stability of a class of switched positive linear time-delay systems," International Journal of Robust and Nonlinear Control, vol. 23, no. 5, pp. 578-589, 2013.

[6] C. Jeong, P. Park, and S. H. Kim, "Improved approach to robust stability and $H_{\infty}$ performance analysis for systems with an interval time-varying delay," Applied Mathematics and Computation, vol. 218, no. 21, pp. 10533-10541, 2012.

[7] B. Zhang, J. Lam, and S. Xu, "Relaxed results on reachable set estimation of time-delay systems with bounded peak inputs," International Journal of Robust and Nonlinear Control, vol. 26, no. 9, pp. 1994-2007, 2016.

[8] O. M. Kwon, M. J. Park, J. H. Park, S. M. Lee, and E. J. Cha, "Improved approaches to stability criteria for neural networks with time-varying delays," Journal of the Franklin Institute. Engineering and Applied Mathematics, vol. 350, no. 9, pp. 27102735, 2013.

[9] W. I. Lee, S. Y. Lee, P. Park, and S. Lee, "Improved criteria on robust stability and performance analysis for systems with interval time-varying delay via new triple integral functionals," Applied Mathematics and Computation, vol. 243, pp. 570-577, 2014.

[10] P. Park, J. W. Ko, and C. Jeong, "Reciprocally convex approach to stability of systems with time-varying delays," Automatica, vol. 47, no. 1, pp. 235-238, 2011.

[11] X.-M. Zhang and Q.-L. Han, "Novel delay-derivativedependent stability criteria using new bounding techniques," International Journal of Robust and Nonlinear Control, vol. 23, no. 13, pp. 1419-1432, 2013.

[12] Y. He, Q.-G. Wang, C. Lin, and M. Wu, "Delay-range-dependent stability for systems with time-varying delay," Automatica, vol. 43, no. 2, pp. 371-376, 2007.

[13] D. Yue, Q.-L. Han, and J. Lam, "Network-based robust $\mathrm{H}_{\infty}$ control of systems with uncertainty," Automatica, vol. 41, no. 6, pp. 999-1007, 2005.

[14] A. Seuret and F. Gouaisbaut, "Wirtinger-based integral inequality: application to time-delay systems," Automatica, vol. 49, no. 9, pp. 2860-2866, 2013.

[15] P. Park, W. I. Lee, and S. Y. Lee, "Auxiliary function-based integral inequalities for quadratic functions and their applications to time-delay systems," Journal of the Franklin Institute, vol. 352, no. 4, pp. 1378-1396, 2015.

[16] L. V. Hien and H. Trinh, "An enhanced stability criterion for time-delay systems via a new bounding technique," Journal of the Franklin Institute. Engineering and Applied Mathematics, vol. 352, no. 10, pp. 4407-4422, 2015.

[17] L. V. Hien and H. Trinh, "Refined Jensen-based inequality approach to stability analysis of time-delay systems," IET Control Theory \& Applications, vol. 9, no. 14, pp. 2188-2194, 2015.

[18] Y. Liu, S. M. Lee, O. M. Kwon, and J. H. Park, "New approach to stability criteria for generalized neural networks with interval time-varying delays," Neurocomputing, vol. 149, pp. 1544-1551, 2015.

[19] T. L. Fernando, V. N. Phat, and H. M. Trinh, "Decentralized stabilization of large-scale systems with interval time-varying delays in interconnections," International Journal of Adaptive Control and Signal Processing, vol. 26, no. 6, pp. 541-554, 2012. 
[20] C.-C. Hua, J. Leng, and X.-P. Guan, "Decentralized MRAC for large-scale interconnected systems with time-varying delays and applications to chemical reactor systems," Journal of Process Control, vol. 22, no. 10, pp. 1985-1996, 2012.

[21] X. Liu and H. Zhang, "Delay-dependent robust stability of uncertain fuzzy large-scale systems with time-varying delays," Automatica, vol. 44, no. 1, pp. 193-198, 2008.

[22] V. N. Phat, N. T. Thanh, and H. Trinh, "Full-order observer design for nonlinear complex large-scale systems with unknown time-varying delayed interactions," Complexity, vol. 21, no. 2, pp. 123-133, 2015.

[23] N. T. Thanh and V. N. Phat, "Decentralized stability for switched nonlinear large-scale systems with interval time-varying delays in interconnections," Nonlinear Analysis: Hybrid Systems, vol. 11, pp. 22-36, 2014.

[24] S. J. Yoo and J. B. Park, "Decentralized adaptive output-feedback control for a class of nonlinear large-scale systems with unknown time-varying delayed interactions," Information Sciences, vol. 186, pp. 222-238, 2012.

[25] X. Zhao, H. Yang, H. R. Karimi, and Y. Zhu, "Adaptive neural control of MIMO nonstrict-feedback nonlinear systems with time delay," IEEE Transactions on Cybernetics, vol. 46, no. 6, pp. 1337-1349, 2015.

[26] H. Liu, Y. Shen, and X. Zhao, "Asynchronous finite-time $H_{\infty}$ control for switched linear systems via mode-dependent dynamic state-feedback," Nonlinear Analysis. Hybrid Systems. An International Multidisciplinary Journal, vol. 8, pp. 109-120, 2013.

[27] G. Chen and Y. Yang, "Finite-time stability of switched positive linear systems," International Journal of Robust and Nonlinear Control, vol. 24, no. 1, pp. 179-190, 2012.

[28] H. Liu, Y. Shen, and X. Zhao, "Finite-time stabilization and boundedness of switched linear system under state-dependent switching," Journal of the Franklin Institute, vol. 350, no. 3, pp. 541-555, 2013.

[29] P. Niamsup, K. Ratchagit, and V. N. Phat, "Novel criteria for finite-time stabilization and guaranteed cost control of delayed neural networks," Neurocomputing, vol. 160, pp. 281-286, 2015.

[30] K. Shi, S. Zhong, H. Zhu, X. Liu, and Y. Zeng, "New delaydependent stability criteria for neutral-type neural networks with mixed random time-varying delays," Neurocomputing, vol. 168, pp. 896-907, 2015.

[31] P. Gahinet, A. Nemirovskii, A. J. Laub, and M. Chilali, LMI Control Toolbox for Use with MATLAB, The MathWorks, Natick, Mass, USA, 1995.

[32] Y. Du, S. Zhong, N. Zhou, L. Nie, and W. Wang, "Exponential passivity of BAM neural networks with time-varying delays," Applied Mathematics and Computation, vol. 221, pp. 727-740, 2013. 


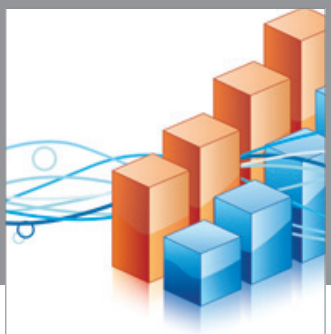

Advances in

Operations Research

vatem alat4

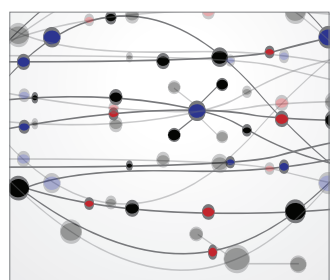

\section{The Scientific} World Journal
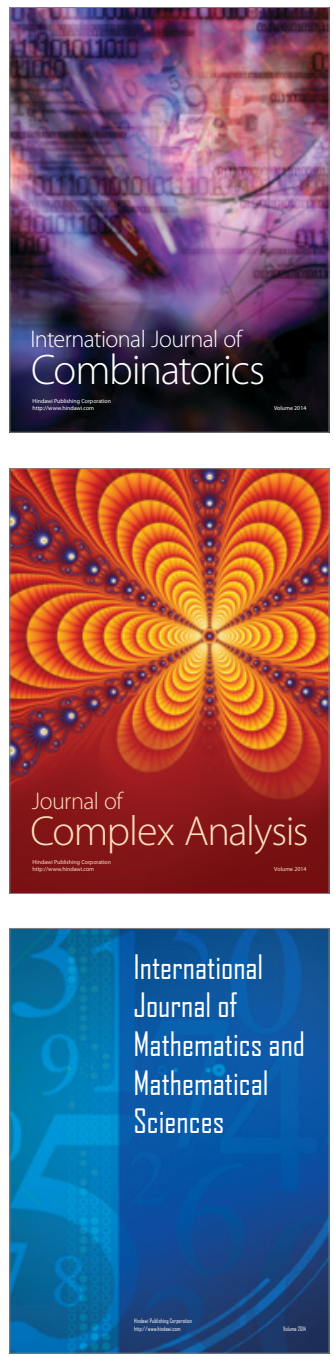
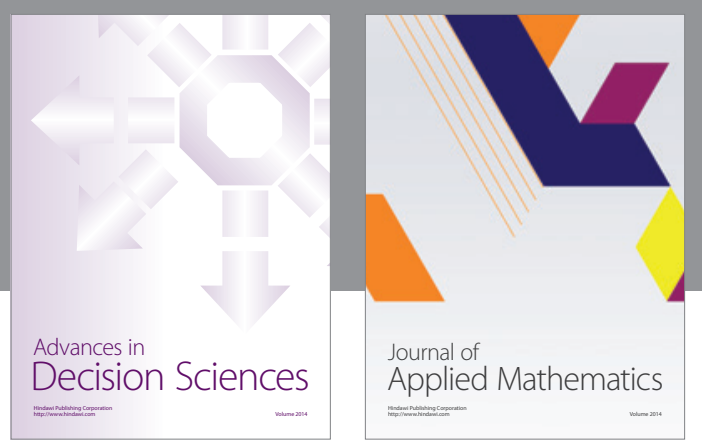

Algebra

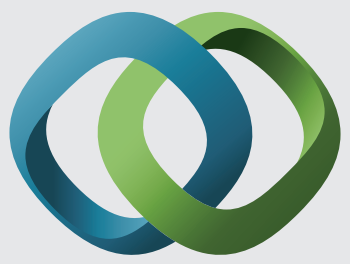

\section{Hindawi}

Submit your manuscripts at

https://www.hindawi.com
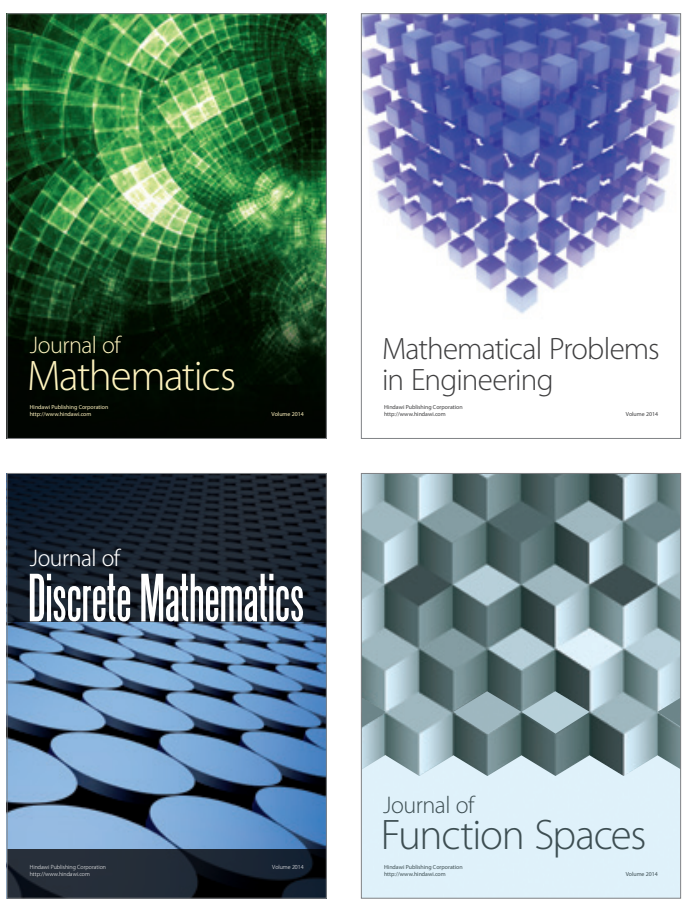

Mathematical Problems in Engineering
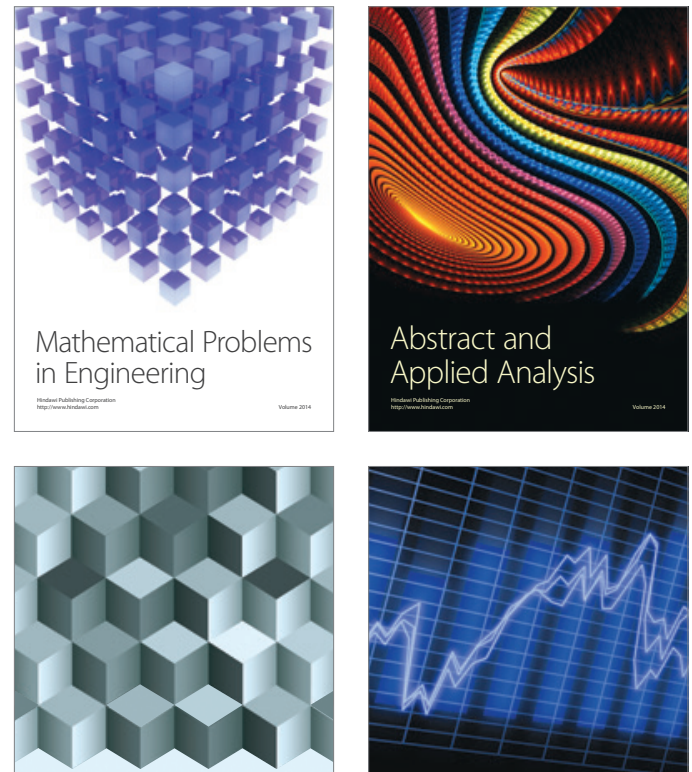

Journal of

Function Spaces

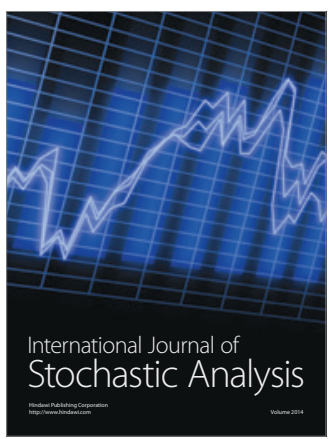

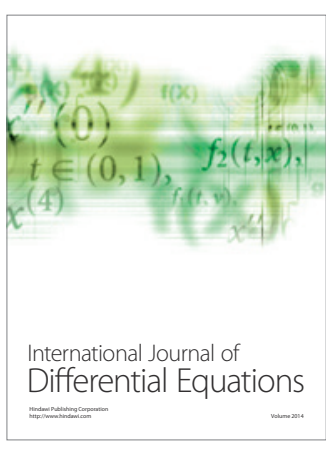
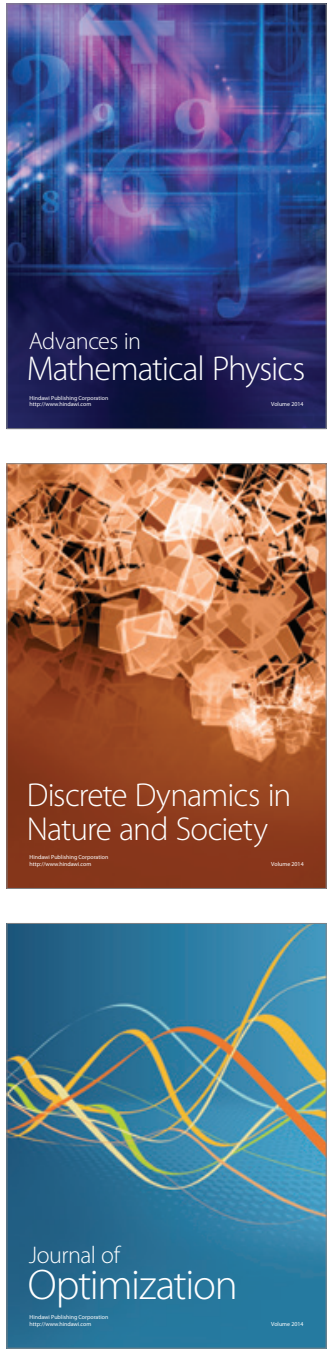